\title{
Automatic Measurement of Garment Using Image Processing
}

\author{
Karunamoorthy Balasubramaniam, J. Ramprabu
}

\begin{abstract}
In this paper we can employ automatic garment size measuring in textile industry. Textile industry is necessary and wants to be advanced in manufacturing of garments without defects. So that measuring the sizes of garments in industry is still a manual process which affects the precision of measurement. Hence the development of machine vision in image processing technology is used. To improve the automatic measurement a switching median filter is used. It remove the noises in the images. After that boundarylocalization algorithm is used to detect the boundaries of the garment. Then K-means clustering algorithm based edge detection helps to measure the number of pixels occupied in the garment and size of garment is measured from the count of pixels. This garment size measurement is implemented in MATLAB 2014a.
\end{abstract}

Keywords : Switching median filter, boundary localization.

\section{INTRODUCTION}

Material industry should be progressed in assembling of articles of clothing without imperfections. In any case, estimating the extents of pieces of clothing in industry is as yet a manual procedure which influences the estimation of article of clothing. Estimating the sizes by manual procedure which additionally takes part of time. In addition to diminish the estimation blunder, administrators need to rehash the task a few times. The precision and proficiency of the estimations are both constrained in customary manner. Numerous individuals displayed a strategy for estimating piece of clothing by recognizing key purposes of the articles of clothing. Estimations are for the most part dependent on corners and centroid of the articles of clothing .Besides, deviation of the camera will make the estimation mistaken.

\section{II.PROPOSED METHOD BLOCK DIAGRAM}

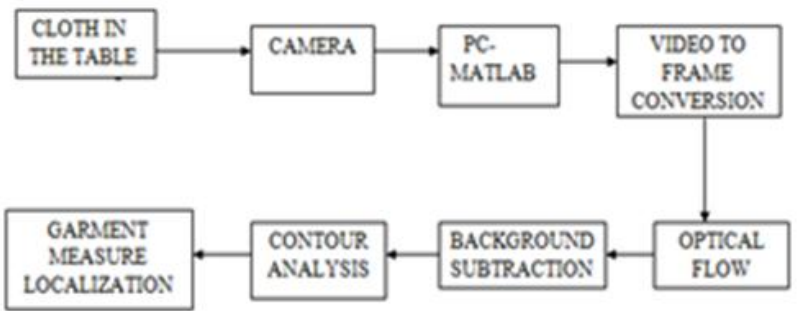

Fig. 1 BLOCK DIAGRAM

\section{III.COMPONENTS}

\section{1 )DIGITAL CAMERA:}

Revised Manuscript Received on 14 August, 2019.

KarunamoorthyBalasubramaniam, Associate professor, Electrical andElectronicsEngineering, Kumaraguru college of Technology, Coimbatore, Tamilnadu, India.(Email: karunamoorthy.b.eee@kct.ac.in)

J. Ramprabu, AssistantProfessor, Electrical andElectronicsEngineering, Kumaraguru college of Technology, Coimbatore, Tamilnadu, India.(Email: ramprabu.j.eee@kct.ac.in)
Considering the strength of the shooting stand, the camera is fixed toward the finish of a pillar. Standard EOS 100D cameras is chosen for the accompanying reasons. Its light weight advances to our desires. Additionally, it gives an Application Programming Interface (API) which can be utilized for programming to control the camera by the PC. Furthermore, it has great execution on adjusting the goals proportion and light affectability. For instance, its APS Csensor makes pictures considerably more stunning and top quality. Correspondingly, a DIGIC 5 processor causes the camera to have great capacity of commotion opposition even in the night mode. After effects of tests showed that photos taken by the camera 100D have superb shading execution, which demonstrates this kind of the camera can absolutely meet the prerequisites for shooting.

\section{SOFTWARE DESCRIPTION}

MATLAB:

Matlab is an elite programming for specialized figuring. It is anything but difficult to utilize condition for calculation, perception and programming. The utilizations are Math and calculation, Algorithm advancement, displaying, reproduction and prototyping.

\section{METHODOLOGY}

In Pre-Processing Step our picture will change over into shading picture to dark scale picture. In Morphological task is perform with the end goal of fragment our picture in square and white area so for effectively recognizable proof of our info picture.

\section{MORPHOLOGICAL TRANSFORMATIONS \\ - EROSION \\ - DILATION}

EROSION:

\begin{tabular}{|l|l|l||l|l|l|l|l|}
\hline 1 & 1 & 1 & 1 & 1 & 1 & 1 & 1 \\
\hline 1 & 1 & 1 & 1 & 1 & 1 & 1 & 1 \\
\hline 1 & 1 & 1 & 1 & 1 & 0 & 1 & 1 \\
\hline 0 & 0 & 1 & 1 & 1 & 0 & 1 & 1 \\
0 & 1 & 1 & 0 & 0 & 0 & 0 & 1 \\
\hline 1 & 1 & 1 & 0 & 0 & 1 & 1 & 1 \\
\hline 1 & 1 & 0 & 0 & 1 & 1 & 1 & 1 \\
\hline
\end{tabular}

Fig.2 Erosion

Published By:

Blue Eyes Intelligence Engineering

\& Sciences Publication 


\section{DILATION}

\begin{tabular}{|l|ll|l|l|l|l|l|}
$\mathbf{0}$ & $\mathbf{0}$ & $\mathbf{0}$ & $\mathbf{0}$ & 1 & 1 & 1 & 1 \\
$\mathbf{0}$ & $\mathbf{0}$ & $\mathbf{0}$ & $\mathbf{0}$ & 1 & 1 & 1 & 1 \\
$\mathbf{0}$ & $\mathbf{0}$ & 1 & 1 & $\mathbf{0}$ & $\mathbf{0}$ & 1 & 1 \\
\hline $\mathbf{0}$ & $\mathbf{0}$ & 1 & $\mathbf{0}$ & $\mathbf{0}$ & $\mathbf{0}$ & 1 & 1 \\
\hline $\mathbf{0}$ & 1 & 1 & $\mathbf{0}$ & $\mathbf{0}$ & $\mathbf{0}$ & $\mathbf{0}$ & 1 \\
\hline 1 & 1 & 1 & $\mathbf{0}$ & $\mathbf{0}$ & 1 & 1 & 1 \\
\hline 1 & 1 & $\mathbf{0}$ & $\mathbf{0}$ & 1 & 1 & 1 & 1 \\
\hline
\end{tabular}

Fig.3 Dilation

\section{VII.IMPLEMENTATION}

\section{- $\quad$ Switching Median Filter}

- Boundary Localization

- $\quad$ K-means Clustering based Edge detection

\section{K means Clustering}

Group investigation, a significant innovation in information mining, is a compelling technique for examining and finding valuable data from various information. $\mathrm{T}$ he bunch calculation bunches the information into classes. So questions inside a group have high likeness in contrast with each other, yet are exceptionally not at all like items in different bunches . Regularly, separation measures are utilized. As a part of measurements and a case of unaided picking up, grouping gives us an accurate and unpretentious examination apparatus from the mathematic see K-implies calculation has a place with a well known segment technique in bunch investigation. The most broadly utilized grouping mistake standard is squared-blunder foundation, it very well may be characterized as

$$
J_{c}=\sum_{j=1}^{c} \sum_{k=1}^{n_{j}}\left\|x_{k}^{(j)}-m_{j}\right\|^{2}
$$

where $\mathbf{J}$, is the total of square-blunder for all items in the database, $\mathrm{xk}$ is the point in space speaking to a given article, and $\mathrm{mj}$ is the mean of bunch cj .Receiving the squaredmistake basis, K-implies functions admirably when the bunches are conservative mists that are fairly all around isolated from each other and are not appropriate for finding groups with non convex shapes or groups of altogether different size. For endeavouring to limit the square-emor standard, it will isolate the articles in a single group into at least two bunches. Notwithstanding that, when applying this square-mistake measure to assess the grouping results, the ideal bunch compares to the radicalism. Since the target capacity has numerous nearby negligible qualities, if the consequences of instatement are actually close to the neighbourhood insignificant point, the calculation will end at a nearby ideal. Along these lines, arbitrary choosing beginning bunch focus is anything but difficult to get in the neighbourhood ideal not the whole ideal. For defeating that square-blunder standard is difficult to recognize the huge contrast among the bunches, one procedure has been created which depends on delegate point-based system. In addition, there are different ways to deal with taking care of the issue that the exhibition of calculation vigorously relies upon the underlying beginning conditions: the least complex one is redundancy with various arbitrary determinations a few calculations likewise utilize reproduction temper strategy to abstain from getting into nearby ideal. The thought is that different sub-tests are drawn from the dataset grouped freely, at that point these arrangements are bunched again individually, the refined beginning focus is then picked as the arrangement having insignificant mutilation over all arrangements. Going for the reliance to introductory conditions and the impediment of $\mathrm{K}$-implies calculation that applies the square-mistake rule to quantify the nature of bunching, this paper displays another improved K-implies calculation that depends on successful procedures of multiinspecting and once-grouping to look through the ideal starting estimations of group focuses. Our trial results show the new calculation can acquire better security and exceed expectations the first $\mathrm{K}$-implies in bunching results.

\section{VIII.ALGORITHM}

The Euclidean separation is utilized in this paper. The separation between one vector $\mathrm{X}=(\mathrm{xl}, \mathrm{x} 2, \ldots, \mathrm{xn})$ and the other vector $\mathrm{Y}=(\mathrm{yl}, \mathrm{y} 2, \ldots, \mathrm{y} \mathrm{n})$ is depicted as pursues.

The separation between an information point $X$ and an information point $\mathrm{d}(\mathrm{X}, \mathrm{V})=\min (\mathrm{d}(\mathrm{X}, \mathrm{Y}), \mathrm{Y} E \mathrm{~V})$

Assume there are $\mathrm{n}$ information focuses in the populace $\mathrm{U}$ and we need to segment $\mathrm{U}$ into $\mathrm{k}$ classes. Set $\mathrm{m}=\mathrm{l}$. At that point the calculation is depicted as pursues.

(1) Compute removes between every datum point and the majority of different information focuses in U; locate the two information focuses between which the separation is the most brief and structure an information point set $\mathrm{Am}(1<\mathrm{m}<\mathrm{k})$ which contains these two information focuses; erase these two information focuses from $\mathrm{U}$,

(2) Find the information point in $U$ that is nearest to the information point set $\mathrm{Am}$, add it to $\mathrm{Am}$ and erase it from $\mathrm{U}$.

(3) Repeat step (2) till the quantity of information focuses In Am comes to :

(4) If $\mathrm{m}<\mathrm{k}$, at that point $\mathrm{m}=\mathrm{m}+1$; discover another pair of information focuses between which the separation is the most limited in $\mathrm{U}$ and structure another information point set Am and erase them from $\mathrm{U}$ at that point go to step (2)

5) For each $\mathrm{Am}(\mathrm{I}<\mathrm{m}<\mathrm{k})$ total the vectors of information focuses and separate the entirety by the quantity of information focuses in Am, at that point every datum point set yields a vector and we select these vectors as the underlying centroids.

(6) Execute the procedure of the standard k-implies calculation from stage 2 .

The estimation of an is distinctive as to various information. On the off chance that the estimation of an is excessively little, every one of the centroids might be gotten in a similar area that contains numerous comparative information focuses; yet in the event that the estimation of an is too enormous, the centroids may stray away from the locale that contains numerous comparative information focuses. As indicated by the consequences of our experiment,better bunching can frequently be acquired if the estimation of an is set to be 0.75 . 


\section{RESULT AND DISCUSSION}

\subsection{Simulation Results:}

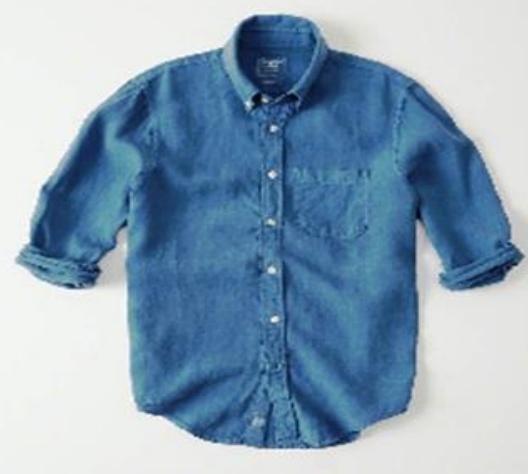

Fig.4 Input Image

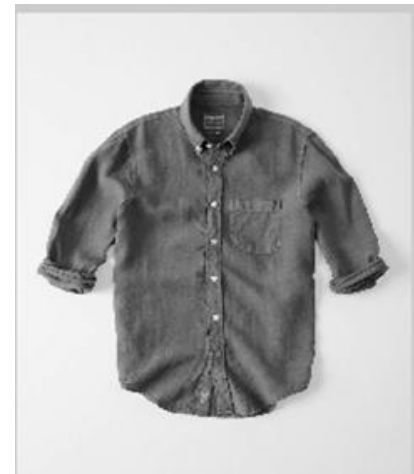

Fig.5 Pre-Processing

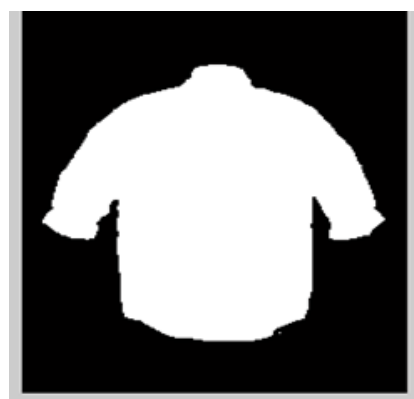

Fig.6 Morphologicaloperation Image

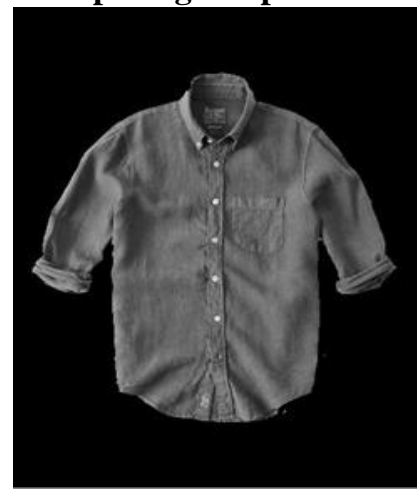

Fig.7 Binarization Image
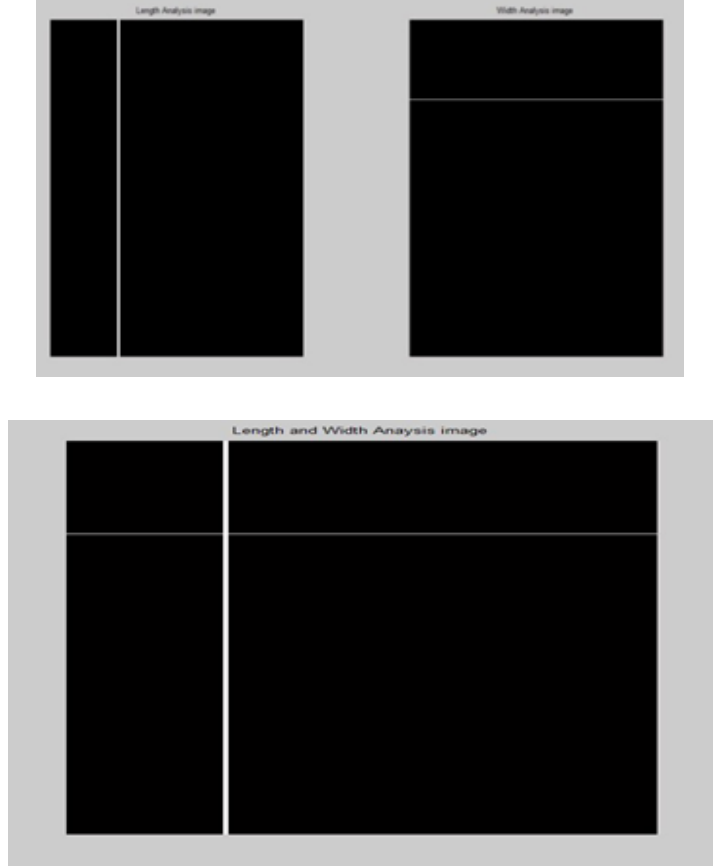

Fig.8 Length Analysis Image

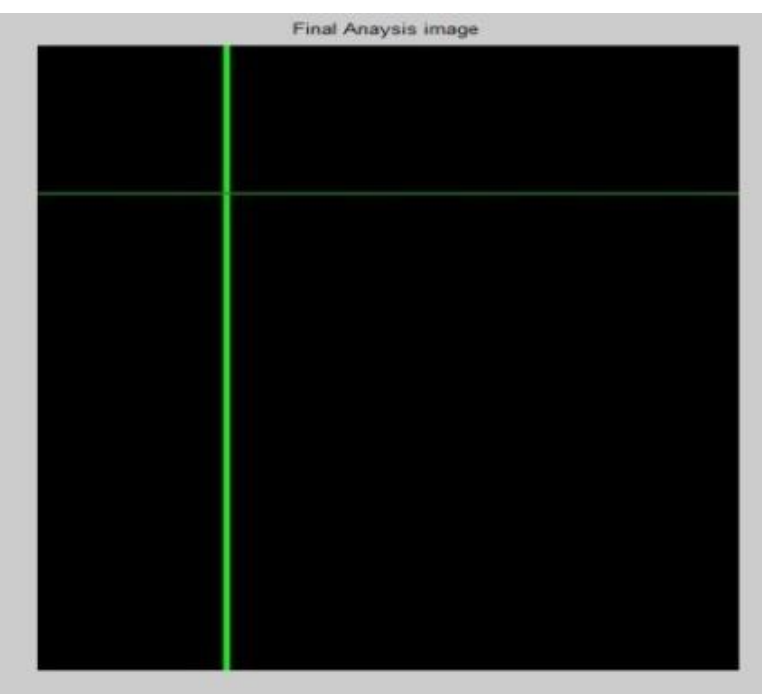

Fig.9 Final Analysis Image

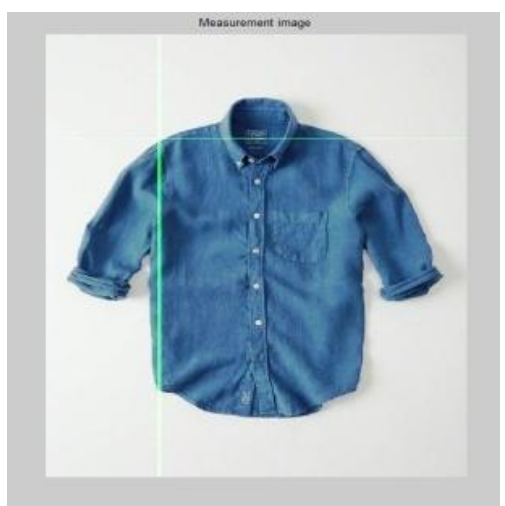

Fig.10 Measurement Image

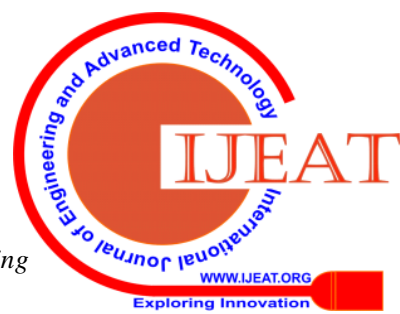




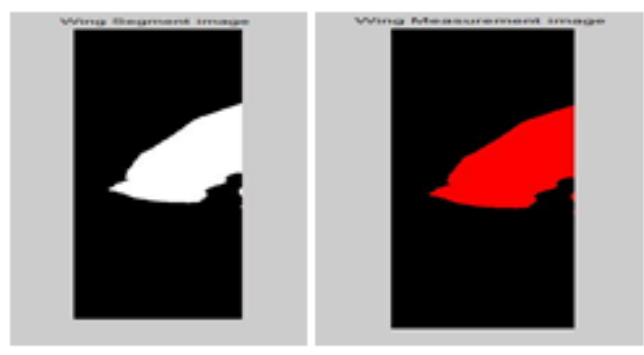

Fig.11 Wing segment imagemeasurement

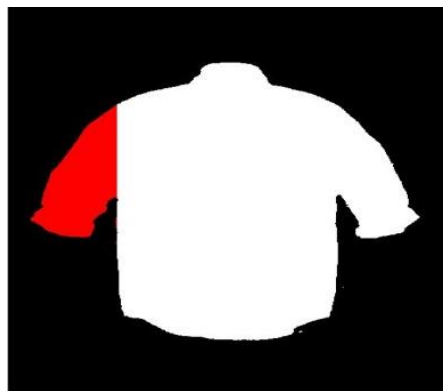

Fig.12Diagram segment image

The actual input image is shown in Fig.4. The preprocessed image of the input is shown in Fig.5. The morphological operation of the input image had shown in Fig.6. The Fig.7 shows the binary image of the input.The Fig.8 shows thelength analysis of the Input image. Final analysis output image shown in Fig.9 output.Figure.10 and Figure.11 shows the measurement image and wing segment image. Fig. 12 shows the diagram of segment image.

\section{OUTPUT:}

Height measurement value: 183.5658

Width measurement value: 183.3466

Wing measurement value: 46.6937

\section{X.FUTURE SCOPE}

Then, there is a lot of space for further improving our strategy. Right off the bat, the internal component focuses are evaluated utilizing an insights strategy. We will abuse an edge distinguishing calculation to look through the inward element focuses naturally and precisely by utilizing the highlights of surfaces and crease lines. Furthermore, it is hard to gauge thick garments precisely utilizing current strategy since the thickness brings an estimation blunder. We will improve the precision of estimation by considering the article of clothing thickness. Finally, we will apply the strategies of AI to perceive article of clothing types and highlight focuses.

\section{XI.CONCLUSION}

In this paper, picture investigation techniques are proposed for programmed estimation of piece of clothing measurements. The switch-middle channel effectively expels indiscreet commotion and non-rash clamor from pictures, and the yield execution is true to form. The limit restriction calculation is reasonable for identifying the edges of the piece of clothing picture and the distinguishing result is effectively and rapidly. K-implies grouping calculation based edge identification estimates the quantity of pixels involved in the piece of clothing and size of the article of clothing is estimated from the tally of pixels. Consequently the size of the piece of clothing is estimated precisely.

\section{REFERENCES}

1. Chunxio Li, Ying Xu, Yi Xiao," Automatic Measurement of Garment Sizes Using Image Recognition" ICGSP '17 Proceedings of the International Conference on Graphics and Signal Processing, Singapore - June 24 - 27, 2017.

2. Li Cao, Yi Jiang, Mingfeng Jiang" Automatic measurement of garment dimensions using machine vision,2010 International Conference on Computer Application and System Modeling (ICCASM 2010).

3. M.O.Ahmad and D.Sundarajan, "Parallel Implementation of a Median Filtering Algorithm," Int. Symp. On Signals and Systems.

4. . X.Xie,R.Sudhakar,andH.Zhuang. "Corner detection by a cost minimization approach",Pattern Recognition. pp.11-24, 1997.

5. B.Karunamoorthy,S.P.SethuD.Somasundareswari, "Fault Detec- tion Using Image Processing Technique for Fabric". Christian College engineering \& technology.

6. R. Srinivasan, K. Anupama, and S. K. Suneeta, [14] FPGA Based ASM implementation for CCD Camera Controller.Indian Institute of,2009.

7. B.karunamoorthy, jayasudhad.somasundareswari, "design and implementation of a system for image based automatic detection and counting of vehicles"," International Journal of Applied Engineering Research.

8. K. T. Gribbon, D. G. Bailey, and A. Bainbridge-Smith, "development issues in using fpgas for image."New:Zealand. Study and Comparison of Various Image Edge Detection Techniques, RamanMaini.

9. Dinesh, B.karunamoorthy, andRaniThottungal ,"Real Time Monitoring System: Implementation of Face Detection and Recognition Algorithm"Vol.8. International Journal of Innovative Technology and Exploring Engineering, PP-404-408,2018. 